Sains Malaysiana 49(3)(2020): 613-624

http://dx.doi.org/10.17576/jsm-2020-4903-16

\title{
Vitamin K Status in Diabetic Patients with Chronic Kidney Disease Stage 3-5 and its Effects on Chronic Kidney Disease - Mineral Bone Disorder
}

(Status Vitamin K pada Pesakit Kencing Manis dengan Kegagalan Ginjal Kronik Tahap 3-5 dan Kesannya kepada Penyakit Ginjal Kronik - Gangguan Mineral Tulang)

\section{S. Jayakumar, Elena AZIZAn, Shamsul Azhar Shah, Arbaiyah Bain \& AbDul Halim Abdul Gafor*}

\section{ABSTRACT}

Diabetic kidney disease (DKD) is common in Malaysia. Vitamin K deficiency among DKD patients may be associated with higher incidence of chronic kidney disease-mineral bone disorder (CKD-MBD). This study was conducted to determine the prevalence of vitamin $K$ deficiency in diabetic patients with $C K D$ stage 3-5. We also correlated vitamin $K$ deficiency with baseline demographic, biochemical results and analysed the effects of vitamin K levels on CKD-MBD. This was a single centre cross-sectional study on diabetic patients with CKD stage 3-5. Demographic profiles were recorded, blood samples were measured for vitamin K level (phylloquinone, proteins induced by vitamin K absence II (PIVKA-II), prothrombin time) and bone markers (parathyroid hormone (PTH), alkaline phosphatase, corrected calcium and phosphate). This study was approved by UKM ethic and research committee (FF-2018-375). Forty-five patients with a median age of 70 (IQR:13) years were recruited. Majority were females (53.3\%) and Malays (64.4\%). Prevalence of patients with insufficient vitamin $K$ was $28.9 \%$ based on the low level of phylloquinone $(<0.4 \mathrm{nmol} / \mathrm{L})$, $93.3 \%$ based on high PIVKA-II level $(>0.66 \mathrm{ng} / \mathrm{mL})$ and $2.2 \%$ based on prolonged prothrombin time $(>14.5 \mathrm{~s})$. PIVKAII was found to be positively correlated with serum creatinine and PTH levels. There were positive correlations between phylloquinone with serum triglyceride and total cholesterol. Prothrombin time was found to be negatively correlated with corrected calcium and total cholesterol. Malay race $(p=0.039)$ and high serum PTH $(p=0.016)$ were significantly associated with higher PIVKA-II concentrations. Multivariable analysis showed serum triglyceride (OR 0.112; CI 95 $\%$ (0.02-0.66); $p=0.017)$ and serum PTH (OR 1.997; CI 95\% (1.01-3.95); $p=0.047)$ were independent predictors for abnormal phylloquinone and PIVKA-II levels, respectively. In conclusion, the prevalence of Vitamin $K$ deficiency was high in diabetic patients with CKD stage 3-5. Serum triglyceride and serum PTH were independent predictor of Vitamin K deficiency.

Keywords: Chronic kidney disease-mineral bone disease; diabetic kidney disease; phylloquinone; protein induced by vitamin Kabsence II (PIVKA II); prothrombin time

\section{ABSTRAK}

Penyakit ginjal yang disebabkan oleh kencing manis (DKD) sangat kerap berlaku di Malaysia. Kekurangan vitamin $K$ dalam kalangan pesakit DKD mungkin berhubung kait dengan kejadian penyakit ginjal kronik-gangguan mineral tulang (CKD-MBD) yang tinggi. Kajian ini dijalankan untuk menentukan kelaziman kekurangan vitamin K pada pesakit DKD tahap 3-5. Kami juga mengkaji hubung kait kekurangan vitamin K dengan demografi dan biokimia asas dan menganalisis kesan paras vitamin K pada CKD-MBD. Ini adalah satu kajian rentas pada pesakit DKD tahap 3-5. Profil demografi direkodkan, sampel darah diambil untuk tahap vitamin $K$ (filokuinon, proteins teraruh dengan ketiadaan vitamin K II (PIVKA-II), tempoh protrombin) dan penanda penyakit CKD-MBD (hormon paratiroid (PTH), fosfatase beralkasi, kalsium dan fosfat). Kajian ini telah diluluskan oleh Jawatankuasa Etika dan Penyelidikan UKM (FF-2018375). Empat puluh lima pesakit dengan umur median 70 (IQR: 13) tahun telah direkrut. Majoriti adalah perempuan $(53.3 \%)$ dan Melayu (64.4\%). Peratusan pesakit dengan kekurangan vitamin K adalah $28.9 \%$ berdasarkan tahap rendah filokuinon $(<0.4 \mathrm{nmol} / \mathrm{L}), 93.3 \%$ berdasarkan tahap PIVKA-II yang tinggi $(>0.66 \mathrm{ng} / \mathrm{mL})$ dan $2.2 \%$ berdasarkan masa protrombin yang berpanjangan (> 14.5 saat). PIVKA-II didapati mempunyai kaitan positif dengan nilai serum kreatinin dan PTH. Terdapat hubung kait positif antara filokuinon dengan serum trigliserida dan kolesterol. Tempoh protrombin didapati berhubung kait secara negatif dengan kalsium dan tahap kolesterol. Bangsa Melayu $(p=0.039)$ dan serum tinggi PTH $(p=0.016)$ adalah berkaitan dengan kepekatan PIVKA-II. Analisis pelbagai pemboleh ubah menunjukkan trigliserida serum (OR 0.112; CI 95\% (0.02-0.66); $p=0.017)$ dan tahap PTH (OR 1.997; CI 95\% (1.013.95); $p=0.047)$ adalah peramal untuk keabnormalan filokuinon dan tahap PIVKA-II . Kesimpulannya, kekurangan vitamin K kerap berlaku pada pesakit diabetes dengan CKD tahap 3-5. Serum trigliserida dan serum PTH adalah peramal bebas kekurangan vitamin $K$.

Kata kunci: Filokuinon; penyakit buah pinggang diabetes; penyakit ginjal kronik - penyakit mineral tulang; protein teraruh dengan ketiadaan vitamin K II (PIVKA II); tempoh protrombin 


\section{INTRODUCTION}

There has been an increasing prevalence of diabetic kidney disease (DKD) in Malaysia and worldwide. DKD is the main cause for ESRD in developed countries ranging from $30-45 \%$ of all cases and also developing countries ranging from $30-60 \%$ of all cases (Ghaderian et al. 2015). Similarly in Malaysia, DKD is the leading cause of renal failure for patients initiating dialysis, increasing from 53 $\%$ in 2004 to $61 \%$ in 2013 (Goh et al. 2014). The prevalence of patients with end stage renal disease (ESRD) on dialysis in Malaysia has increased from 629 per million population in 2007 to 1286 per million population in 2016 (Bujang et al. 2017). This markedly increasing number of ESRD plays an enormous role on human, economic and social burden on our healthcare system.

Patients with DKD has an increased risk for cardiovascular and mineral bone disease. The Clinical Practice Guidelines by The Kidney Disease Improving Global Outcomes (KDIGO) recommends screening patients for the presence of vascular calcification which leads to major complications of the disease (KDIGO 2009) Chronic kidney disease-mineral and bone disorder (CKDMBD) is a systemic disorder impairing the metabolism of bone and minerals in the body via abnormal mineralization and bone turnover which results from impaired calcium, phosphate, parathyroid hormone (PTH) and vitamin D metabolism. Mineral metabolism disorders and dysregulation of endocrine function may result in renal bone disease along with skeletal and extra skeletal complications. Patients with CKD-MBD has higher incidences of vascular calcifications, cardiovascular disease and lower bone mineral contents as the disease progresses (Klawansky et al. 2003). Factors like hyperphosphatemia, vitamin D deficiency, chronic inflammation, increased calcium load may promote calcification (Cozzolino et al. 2019).

Vitamin $\mathrm{K}$ is a fat-soluble vitamin which acts as a co-factor for the enzyme $\gamma$-glutamyl carboxylase (GGCX). It is needed for the activation of vitamin $\mathrm{K}$-dependent proteins (VKDPs) which involved in hemostasis as well as vascular and bone health (Berkner \& Runge 2004). VKDPs which are the osteocalcin and matrix GLA protein protects from bone fractures and cardiovascular calcifications, respectively. Vitamin K recycling system helps to maintain a sufficient level of vitamin $\mathrm{K}$ for activation of VKDPs through GGCX (Berkner \& Runge 2004).

Two forms of vitamin $\mathrm{K}$, which are available can be found in green vegetables known as K1 or phylloquinone, and $\mathrm{K} 2$ or menaquinones, found in specific food (such as natto, in Japan) or derived from the metabolic activity of intestinal bacteria (Booth \& Al Rajabi 2008). An important cause for vitamin $\mathrm{K}$ deficiency in CKD patients is reduction of dietary intake due to patient's uremic symptoms. It has been shown that an adequate amount of vitamin $\mathrm{K}$ intake may help to prevent bone mineral disorders and vascular calcifications (Kaesler et al. 2014).
CKD patients with vitamin K deficiency had higher incidence of cardiovascular disease and bone fractures among the American and Canadian populations in a review by McCabe et al. (2013). Liver is the site of synthesis of vitamin $\mathrm{K}$ dependent coagulation factors and is the major site of storage of vitamin K. Storage of vitamin K can be evaluated by the direct measurement of circulating Vitamin K1 (Booth \& Al Rajabi 2008). Vitamin K deficiency can be analysed via markers such as phylloquinone level $(<$ $0.4 \mathrm{nmol} / \mathrm{L}$ ), proteins induced by vitamin $\mathrm{K}$ absence (PIVKA-II) (>2 nmol/L) (Elliott et al. 2014) and prolonged prothrombin time (PT) (Quick 1975).

In a study conducted by Holden et al. (2010) among 172 stage 3 to 5 CKD patients, more than $50 \%$ of them consumed less than the recommended adequate intake of vitamin $\mathrm{K}$ which was monitored with serum phylloquinone level, osteocalcin and protein induced by vitamin $\mathrm{K}$ absence (PIVKA-II). It was concluded that lower dietary intake of vitamin $\mathrm{K}$ predicted lower levels of phylloquinone (6\% insufficient) and higher levels of PIVKA-II (97\% insufficient). A study done by Kohlmeier et al. (1997) showed that patients with fractures and hyperparathyroidism were more severe in patients with poor vitamin $\mathrm{K}$ status. Patients with low phylloquinone concentrations had severely elevated PTH concentrations. The correlation between vitamin $\mathrm{K}$ and PTH concentrations suggests that a vitamin K-dependent process is important for the regulation of parathyroid hormone.

We hypothesize that Vitamin $\mathrm{K}$ deficiency is common in diabetic patients with CKD stage 3-5 and is associated with higher incidence of CKD-MBD. Thus, we embarked on this study to determine the prevalence of vitamin $\mathrm{K}$ deficiency in diabetic patients with CKD stage 3-5 patients and study its correlation with CKD-MBD.

\section{MATERIALS AND METHODS}

This was a single centre cross-sectional study involving diabetic patients with CKD stage 3-5 receiving nephrology care at Hospital Canselor Tuanku Muhriz (HCTM), Universiti Kebangsaan Malaysia Medical Centre. This study was approved by UKM Ethic and Research Committee (FF-2018-375). We included diabetic patients with CKD stages 3-5 who were more than 18 years old and not on warfarin or novel oral anticoagulants treatment. Our exclusion criteria included patients on renal replacement therapy, vitamin $\mathrm{K}$ supplement for the last 3 months, history of malignancy tumour disease and alcohol or drug abuse, malabsorption syndromes, chronic liver disease, mental condition rendering the subject unable to understand the nature, scope and possible consequences of the study, pregnant and lactating patients, chronic infection, participation in any previous trial on vitamin $\mathrm{K}$, history of non-compliance and unable to provide signed informed consent. Diabetic patients with CKD stage 3-5 (stage 3 eGFR $30-59 \mathrm{~mL} / \mathrm{min} / 1.73 \mathrm{~m}^{2}$, stage 4 eGFR $15-29$ $\mathrm{mL} / \mathrm{min} / 1.73 \mathrm{~m}^{2}$, stage 5 eGFR less than $15 \mathrm{~mL} / \mathrm{min} / 1.73$ 
$\mathrm{m}^{2}$ non dialysis) under the nephrology clinic follow up were enrolled after initial screening. For estimation of Glomerular Filtration Rate (eGFR), Chronic Kidney Disease Epidemiology Collaboration (CKD-EPI) equation was used (Levey et al. 2009). Venous blood were collected for measurement of vitamin $\mathrm{K}$ level (phylloquinone, PIVKA-II, PT) and CKD-MBD markers (PTH, alkaline phosphatase, corrected calcium and phosphate).

Vitamin K1 can be measured using a modified highperformance liquid chromatography (HPLC) method and fluorescence detection (Azharuddin et al. 2007). HPLC with fluorescence detection has shown greater sensitivity and selectivity compared to UV detection but extensive sample pre-purification is required to reduce the chromatographic interference induced by lipid. Detection of human Vitamin K1 using Enzyme-Linked Immunosorbent Assay (ELISA) method has high sensitivity and specificity (Vitamin K1, Amsbio).

Serum PIVKA-II is determined using a monoclonal antibody to PIVKA-II in an ELISA (Belle et al. 1995) This assay has high sensitivity and excellent specificity for detection of human PIVKA-II and no significant crossreactivity or interference between human PIVKA-II and analogues were observed (PIVKA-II, Amsbio).

Serum for phylloquinone and PIVKA II assays were centrifuged at $3000 \mathrm{rpm}$ for $15 \mathrm{~min}$ at room temperature and stored at $-80{ }^{\circ} \mathrm{C}$ before the analysis. Serum phylloquinone and PIVKA II were measured in duplicates using sandwich enzyme immunoassay technique, in a single laboratory and was assayed in a single batch to avoid inter assay variations. Phylloquinone were measured using Human Vitamin K1 ELISA technique (AMS Biotechnology (Europe) Limited, UK). Phylloquinone level (normal range 0.4-3.44 nmol/L) evaluate storage of vitamin $\mathrm{K}$ and nutritional status. A low level of phylloquinone $(<0.4$ $\mathrm{nmol} / \mathrm{L}$ ) indicates vitamin K deficiency (Elliot et al 2014).

PIVKA II concentration (normal range $0.4-0.66 \mathrm{ng} /$ $\mathrm{mL}$ ) were determined by using commercially available kits from AMS Biotechnology which uses Human PIVKA II ELISA technique. PIVKA II is an early marker of subclinical vitamin K deficiency. A raised PIVKA II level $(>0.66 \mathrm{ng} / \mathrm{mL}$ ) indicates vitamin $\mathrm{K}$ deficiency (Elliot et al 2014). PT is an indirect marker of vitamin K deficiency. Prolonged PT (more than 14.5 s) indicates vitamin K deficiency (Quick 1975). PT, corrected calcium, PTH, alkaline phosphatase, and phosphate samples were measured using routine methods available in our centre.

\section{StATISTICAL ANALYSES}

Data were analysed using the Statistical Package for Social Science (SPSS) software version 25. Normally distributed data were expressed as mean \pm standard deviation (SD) and non-normally distributed data were expressed as median (IQR). Correlations were tested using Spearman correlation coefficients. For the categorical variables, frequencies and percentages were obtained and tabulated.
Categorical variables were analysed using Chi squared test or Fisher's Exact test as appropriate. The magnitude of associations was tested using ratios and confidence intervals for the ratios. Multivariate analyses were performed using binary logistic regression to determine independent predictors of vitamin K status. All statistical tests were two sided and an unadjusted $\mathrm{p}$ value of $<0.05$ was considered statistically significant.

\section{RESULTS}

\section{DEMOGRAPHY}

The demographic and clinical details of all the patients are shown in Table 1. There were forty-five patients with a median age of 70.0 (IQR:13.0) years were recruited. Majority were females (53.3\%) and Malays (64.4\%). Based on comorbidities, $82.2 \%$ had hypertension, $66.7 \%$ had dyslipidaemia, $15.6 \%$ with ischemic heart disease and $8.9 \%$ had history of cerebrovascular disease.

\section{PHYLLOQUINONE}

Thirteen patients $(28.9 \%)$ met criteria for vitamin $\mathrm{K}$ deficiency based on the low level of phylloquinone $(<0.4$ $\mathrm{nmol} / \mathrm{L})$. Overall median serum phylloquinone concentration in 45 patients was $0.53(\mathrm{IQR}: 0.72) \mathrm{nmol} / \mathrm{L}$ (Table 1). There were positive correlations between phylloquinone and serum triglyceride $(\mathrm{p}=0.002)$ and total cholesterol $(\mathrm{p}=0.006)$ (Table 2$)$. High serum triglyceride (OR 8.06; CI 95\% (1.52-41.7); $\mathrm{p}=0.007)$ and total cholesterol (8.12; CI 95\% (1.02-71.4); $\mathrm{p}<0.038)$ levels were strongly associated with higher phylloquinone concentrations. We found no strong correlation and significant association between phylloquinone concentration and CKD-MBD markers (PTH, alkaline phosphatase, corrected calcium and phosphate) (Table 3 ). The single independent predictor of abnormal phylloquinone concentration was serum triglycerides (OR 0.112; CI 95\% (0.02-0.66); $\mathrm{p}=0.017$ ) (Table 4).

\section{PROTEINS INDUCED BY VITAMIN K ABSENCE II (PIVKA-II)}

The median serum PIVKA-II level measured in 45 patients was 2.23 (IQR: 1.94) ng/mL. There were $93.3 \%$ of patients had vitamin K deficiency based on high PIVKA-II level $>0.66 \mathrm{ng} / \mathrm{mL}$, corresponding to worse vitamin $\mathrm{K}$ status (Table 1). PIVKA-II was found to be positively correlated with serum creatinine $(\mathrm{p}=0.032)$ and PTH $(\mathrm{p}=0.005)$ levels (Table 2). Malay race (OR 1.23; CI 95\% (1.01-1.56); $\mathrm{p}=0.039)$ and high serum PTH (OR 1.33; CI $95 \%$ (1.02 - 1.85); $\mathrm{p}=0.016)$ were significantly associated with higher PIVKA-II concentrations. There was no significant correlation between PIVKA II concentration and other CKD-BMD markers such as alkaline phosphatase, corrected calcium and phosphate (Table 3 ). The only independent predictor of abnormal PIVKA-II concentration 
TABLE 1. Demographic and clinical features of study population

\begin{tabular}{|c|c|c|}
\hline Variables & $\mathrm{N}(\%)$ & Median (IQR) Mean \pm SD \\
\hline \multicolumn{3}{|c|}{ Clinical characteristics $(n=45)$} \\
\hline Age, years & & $70(13)$ \\
\hline Gender (female) & $24(53.3)$ & \\
\hline \multicolumn{3}{|l|}{ Race } \\
\hline Malay & $29(64.4)$ & \\
\hline Chinese & $16(35)$. & \\
\hline Indian & - & \\
\hline Others & - & \\
\hline $\mathrm{eGFR}, \mathrm{mL} / \mathrm{min}$ per $1.73 \mathrm{~m}^{2}$ & & $21.2(21.1)$ \\
\hline CKD stage 3 & $15(33.3)$ & \\
\hline CKD stage 4 & $15(33.3)$ & \\
\hline CKD stage 5 & $15(33.3)$ & \\
\hline \multicolumn{3}{|l|}{ Comorbidities } \\
\hline Hypertension & $37(82.2)$ & \\
\hline Cerebrovascular disease & $4(8.9)$ & \\
\hline Ischemic heart disease & $7(15.6)$ & \\
\hline Dyslipidemia & $30(66.7)$ & \\
\hline \multicolumn{3}{|l|}{ Vitamin K status } \\
\hline Phylloquinone, nmol/1 & $13(28.9) *$ & $0.53(0.72)$ \\
\hline PIVKA II, ng/mL & $42(93.3) *$ & $2.23(1.94)$ \\
\hline Prothrombin time, (s) & $1(2.2) *$ & $13.0(1.0)$ \\
\hline \multicolumn{3}{|l|}{ Laboratory measures } \\
\hline Phosphate, $\mathrm{mmol} / \mathrm{l}$ & & $1.16(0.36)$ \\
\hline Corrected calcium, mmol/1 & & $2.41 \pm 0.12$ \\
\hline Serum iPTH, pmol/1 & & $9.8(8.38)$ \\
\hline Serum ALP, $\mathrm{u} / \mathrm{L}$ & & $84.0(39)$ \\
\hline Serum Albumin, g/l & & $36.0(5)$ \\
\hline Serum creatinine, umol/1 & & $231.5(180.3)$ \\
\hline Haemoglobin, g/dl & & $11.91 \pm 1.70$ \\
\hline Serum TG, mmol/1 & & $1.84 \pm 0.73$ \\
\hline Serum TC, mmol/1 & & $4.4(1.19)$ \\
\hline Serum HDL, mmol/1 & & $1.09 \pm 0.28$ \\
\hline Serum LDL, mmol/1 & & $2.32(1.34)$ \\
\hline Hbalc, $\%$ & & $7.5(2.75)$ \\
\hline
\end{tabular}

*Vitamin K deficiency. Normally distributed data were expressed as mean $\pm(\mathrm{SD})$ and non-normally distributed data were expressed as median (IQR). eGFR, estimated glomerular filtration rate; CKD, chronic kidney disease; PIVKA II, proteins induced by vitamin K absence; PTH, parathyroid hormone; ALP, alkaline phosphatase; TG, triglycerides; TC, total cholesterol; HDL, high density lipoprotein; LDL, low density lipoprotein

by binary logistic regression was serum PTH (OR 1.997; CI 95\% (1.01-3.95); $\mathrm{p}=0.047)$ (Table 4).

\section{PROTHROMBIN TIME (PT)}

Median PT in 45 patients was 13.0 (1.0) s. Vitamin K deficiency criteria defined as $>14.5 \mathrm{~s}$ was met in one patient (2.2\%). PT was found to be negatively correlated with corrected calcium $(\mathrm{p}=0.048)$ and total cholesterol $(\mathrm{p}=$ 0.025 ) levels (Table 2). There were no significant associations between prolonged PT and demography, comorbidities and laboratory measurements.

\section{DISCUSSION}

This was the first local study assessing vitamin K status in diabetic patients with CKD stage 3-5 and confirmed that these patients have a poor vitamin $\mathrm{K}$ status. We found that Malay race was significantly associated with higher PIVKA-II concentrations that suggested vitamin K deficiency. As a multicultural country, each race in Malaysia has their own cuisine and this finding may suggest that Malay race did not consume the recommended amount of vitamin $\mathrm{K}$. Lower vitamin $\mathrm{K}$ intake may be related to the dietary restriction recommended in $\mathrm{CKD}$ 
TABLE 2. Spearman Correlations among measures of vitamin K status, demographic and biochemical variables

\begin{tabular}{lcccccc}
\hline Variables & \multicolumn{2}{c}{ Phylloquinon $(\mathrm{nmol} / \mathrm{l})$} & \multicolumn{2}{c}{ PIVKA II $(\mathrm{ng} / \mathrm{mL})$} & \multicolumn{2}{c}{ Prothrombin time $(\mathrm{s})$} \\
& $\mathrm{r}$ & $\mathrm{p}$ & $\mathrm{R}$ & $\mathrm{p}$ & $\mathrm{r}$ & $\mathrm{p}$ \\
\hline Age, years & 0.12 & 0.432 & 0.00 & 0.986 & 0.12 & 0.439 \\
eGFR, mL/min per $1.73 \mathrm{~m}^{2}$ & -0.21 & 0.421 & -0.25 & 0.100 & -0.20 & 0.178 \\
Phylloquinone (nmol/l) & - & - & 0.09 & 0.554 & -0.22 & 0.155 \\
PIVKA II (ng/mL) & 0.09 & 0.554 & - & - & -0.11 & 0.463 \\
Prothrombin time (s) & -0.22 & 0.155 & -0.11 & 0.463 & - & - \\
Phosphate (mmol/l) & 0.08 & 0.583 & -0.10 & 0.511 & 0.02 & 0.902 \\
Corrected calcium (mmol/l) & 0.09 & 0.572 & -0.02 & 0.897 & -0.30 & $0.048^{*}$ \\
Serum PTH (pmol/l) & 0.26 & 0.081 & 0.42 & $0.005^{*}$ & 0.04 & 0.778 \\
Serum ALP (u/l) & 0.81 & 0.243 & 0.14 & 0.375 & -0.01 & 0.938 \\
Serum Albumin (g/l) & 0.10 & 0.532 & 0.22 & 0.142 & -0.24 & 0.112 \\
Serum Creatinine (umol/l) & 0.12 & 0.448 & 0.32 & $0.032^{*}$ & 0.18 & 0.249 \\
Haemoglobin (g/dl) & -0.09 & 0.545 & -0.02 & 0.886 & -0.10 & 0.518 \\
Serum TG (mmol/l) & 0.46 & $0.002^{*}$ & 0.03 & 0.833 & -0.10 & 0.529 \\
Serum TC (mmol/l) & 0.41 & $0.006^{*}$ & 0.13 & 0.382 & -0.34 & $0.025^{*}$ \\
Serum HDL (mmol/l) & 0.16 & 0.306 & 0.10 & 0.515 & -0.13 & 0.400 \\
Serum LDL (mmol/l) & -0.21 & 0.170 & 0.02 & 0.900 & -0.13 & 0.403 \\
Hbalc (\%) & -0.04 & 0.798 & -0.02 & 0.886 & -0.10 & 0.512 \\
\hline
\end{tabular}

* Significant correlation $\mathrm{P}<0.05$; CKD, chronic kidney disease; PIVKA II, proteins induced by vitamin K absence; PTH, parathyroid hormone; ALP, alkaline phosphatase; TG, triglycerides; TC, total cholesterol; HDL, high density lipoprotein; LDL, low density lipoprotein

patients which limits intake of sodium and potassium that subsequently leads to reduced intake of foods with high vitamin $\mathrm{K}$ content as supported by other studies (Beto et al. 2014; Cranenburg et al. 2012). Hence, a larger local study with proper dietary information with food questionnaire needed to confirm the present results. There were more female in our study as supported by other study that showed that diabetic kidney disease was more common in female (Abougalambou et al. 2016) and higher risk of fracture especially diabetic postmenopausal women (Abdulameer et al. 2018). Collins and Cashman (2006) and Tsugawa et al. (2006) reported that elderly population were more susceptible for lower vitamin $\mathrm{K}$ intakes and the circulating uncarboxylated osteocalcin concentrations were highest in the oldest group that clearly indicates the requirement for vitamin $\mathrm{K}$ increase with age respectively. Nevertheless, we did not find any correlation between older age and vitamin $\mathrm{K}$ deficiency. Our finding is similar with a study done by Ikeda et al. (2006) and Yan et al. (2004) which demonstrated that older individuals in northern China and postmenopausal women in Japan have a better vitamin $\mathrm{K}$ status due to higher consumption of green vegetables and natto. As we know, the incidence of osteoporosis related fractures among the older generation is very common due to low bone mass and microarchitectural deterioration of bone tissue (Khir et al. 2012; Pillai et al. 2011). Previous study showed that high vitamin K consumption was associated with reduced bone loss and fracture incidence in elderly people (Cockayne et al. 2006).
The data of this study showed the prevalence of hypertension, dyslipidaemia, ischemic heart disease, cerebrovascular disease at 82.2, 66.7, 15.6 and $8.9 \%$, respectively. Hypertension and dyslipidaemia are major independent risk factor for the development and progression of DKD (Forbes \& Cooper 2013). Study has shown the combination of low vitamin D and $\mathrm{K}$ status was associated with increased blood pressure and a trend for greater hypertension risk (van Ballegooijen et al. 2017). The prevalence of vitamin $\mathrm{K}$ deficiency in CKD patients has shown higher incidences of cardiovascular disease and vitamin $\mathrm{K}$ replacement in $\mathrm{CKD}$ patients protect from cardiovascular calcifications (Gallieni \& Fusaro 2014; Krueger et al. 2009). There is no association between intake of vitamin $\mathrm{K}$ and stroke risk among the cerebrovascular disease population (Vissers et al. 2013). A study by Larsson et al. (2018) demonstrated that genetically higher circulating vitamin K1 levels are associated with an increased risk of large artery atherosclerotic stroke.

In our study, vitamin $\mathrm{K}$ status was investigated using measurements of phylloquinone, PIVKA II and PT. Vitamin $\mathrm{K}$ insufficiency in diabetic patients with CKD stage 3-5 patients was $28.9 \%$ based on low level of phylloquinone. Holden et al. (2010) in a study of 172 stage 3-5 CKD patients, demonstrated that $6 \%$ were vitamin K-insufficient according to low levels of serum phylloquinone. It should be noted, however, that phylloquinone was measured with HPLC test whereas in our study ELISA technique was used. Their results may, therefore, not be comparable with our results. Marinova 
TABLE 3. Association between vitamin K status and socio-demographic factors, comorbidities and laboratory measurements ( $\mathrm{n}=45$ )

\begin{tabular}{|c|c|c|c|c|c|c|c|c|c|}
\hline \multirow[t]{2}{*}{ Socio-demographic } & \multicolumn{3}{|c|}{ Abnormal Phylloquinone $(<0.4 \mathrm{nmol} / \mathrm{L}) \mathrm{P}$} & \multicolumn{3}{|c|}{ Abnormal PIVKA II $(>0.66 \mathrm{ng} / \mathrm{mL})$} & \multicolumn{3}{|c|}{ Abnormal Prothrombin time $(>14.5 \mathrm{~s})$} \\
\hline & $\mathrm{n}(\%)$ & OR $(95 \% \mathrm{CI})$ & $\mathrm{p}$ & $\mathrm{n}(\%)$ & OR $(95 \% \mathrm{CI})$ & $\mathrm{p}$ & $\mathrm{n}(\%)$ & OR $(95 \% \mathrm{CI})$ & $\mathrm{p}$ \\
\hline \multicolumn{10}{|l|}{$\operatorname{Sex}$} \\
\hline Female & $7(29.2)$ & $0.97(0.27-3.54)$ & $0.965^{*}$ & $22(91.7)$ & $1.82(0.15-21.62)$ & 0.99 & $1(4.2)$ & $0.96(0.88-1.04)$ & 0.99 \\
\hline Male & $6(28.6)$ & & & $20(95.2)$ & & & $0(0)$ & & \\
\hline \multicolumn{10}{|l|}{ Age } \\
\hline Below 65 & $4(25.0)$ & $1.4(0.34-5.36)$ & 0.743 & $15(93.8)$ & $0.90(0.08-10.77)$ & 0.99 & $0(0)$ & $1.04(0.97-1.11)$ & 0.99 \\
\hline 65 and above & $9(31)$ & & & $27(93.1)$ & & & $1(3.4)$ & & \\
\hline \multicolumn{10}{|l|}{ Ethnic } \\
\hline Malay & $9(31)$ & $0.74(0.18-2.94)$ & 0.743 & $29(100)$ & $1.23(1.01-1.56)$ & 0.039 & $0(0)$ & $1.07(0.94-1.21)$ & 0.36 \\
\hline Chinese & $4(25)$ & & & $13(81.3)$ & & & $1(6.3)$ & & \\
\hline \multicolumn{10}{|l|}{ Comorbidities } \\
\hline \multicolumn{10}{|l|}{ Hypertension } \\
\hline Yes & $9(24.3)$ & $3.13(0.64-14.29)$ & 0.202 & 34 (91.9) & $0.92(0.83-1-01)$ & 0.99 & $1(2.7)$ & $1.03(0.97-1.08)$ & 0.99 \\
\hline No & $4(50)$ & & & $8(100)$ & & & $0(0)$ & & \\
\hline \multicolumn{10}{|l|}{$C V D$} \\
\hline Yes & $13(28.9)$ & $1.23(0.12-12.50)$ & 0.99 & $3(75)$ & $6.67(0.45-90.90)$ & 0.249 & $0(0)$ & $0.98(0.93-1.02)$ & 0.99 \\
\hline No & $12(29.3)$ & & & $39(95.1)$ & & & $1(2.4)$ & & \\
\hline \multicolumn{10}{|l|}{$I H D$} \\
\hline Yes & $2(25)$ & $1.27(0.22-7.14)$ & 0.99 & $7(87.5)$ & $2.5(0.20-31.25)$ & 0.452 & $0(0)$ & $0.97(0.92-1.03)$ & 0.99 \\
\hline No & $11(29.7)$ & & & 35 (94.6) & & & $1(6.7)$ & & \\
\hline \multicolumn{10}{|l|}{ Dyslipidaemia } \\
\hline Yes & $11(36.7)$ & $0.27(0.05-1.41)$ & 0.165 & $29(36.7)$ & $0.22(0.02-2.70)$ & 0.254 & $0(0)$ & $0.97(0.82-1.07)$ & 0.33 \\
\hline No & $2(13.3)$ & & & $13(86.7)$ & & & $1(6.7)$ & & \\
\hline \multicolumn{10}{|l|}{ Vitamin $K$ status } \\
\hline \multicolumn{10}{|l|}{ Phylloquinone } \\
\hline Abnormal (low) & - & - & - & $12(92.3)$ & $1.25(0.10-14.29)$ & 0.99 & $0(0)$ & $1.03(0.97-1.10)$ & 0.99 \\
\hline Normal & - & - & - & $30(93.8)$ & & & $1(3.1)$ & & \\
\hline
\end{tabular}




\begin{tabular}{|c|c|c|c|c|c|c|c|c|c|}
\hline \multicolumn{10}{|l|}{ PIVKA II } \\
\hline Abnormal (high) & $12(28.6)$ & $1.25(0.10-14.29)$ & 0.99 & - & - & - & $0(0)$ & $1.49(0.68-3.33)$ & 0.07 \\
\hline Normal & $1(33.3)$ & & & - & - & - & $1(33.3)$ & & \\
\hline \multicolumn{10}{|l|}{ Prothrombin time } \\
\hline Abnormal (prolonged) & $13(29.5)$ & $0.71(0.58-0.85)$ & 0.99 & $0(0)$ & $20(5.56-8.33)$ & 0.067 & - & - & - \\
\hline Normal & $0(0)$ & & & $42(95.5)$ & & & - & - & - \\
\hline \multicolumn{10}{|l|}{ Laboratory measures } \\
\hline \multicolumn{10}{|l|}{ Phosphate, mmol/l } \\
\hline Abnormal (High) & $0(0)$ & $1.47(1.19-1.82)$ & 0.308 & $4(100)$ & $1.08(0.99-1.18)$ & 0.99 & $0(0)$ & $1.02(0.98-1.08)$ & 0.99 \\
\hline Normal & $13(31.7)$ & & & $38(92.7)$ & & & $1(2.4)$ & & \\
\hline \multicolumn{10}{|l|}{ Calcium, $\mathrm{mmol} / \mathrm{l}$} \\
\hline Abnormal (Low) & $0(0)$ & $1.41(1.18-1.72)$ & 0.99 & $0(0)$ & $1.08(0.99-1.16)$ & 0.99 & $0(0)$ & $1.02(0.98-0.08)$ & 0.99 \\
\hline Normal & $13(29.5)$ & & & $41(93.2)$ & & & $1(2.3)$ & & \\
\hline \multicolumn{10}{|l|}{ Serum PTH, pmol/l } \\
\hline Abnormal (High) & $9(27.3)$ & $1.33(0.32-5.56)$ & 0.721 & $33(100)$ & $1.33(1.02-1.85)$ & 0.016 & $0(0)$ & $1.08(0.92-1.30)$ & 0.25 \\
\hline Normal & $4(33.3)$ & & & $9(75)$ & & & $1(8.3)$ & & \\
\hline \multicolumn{10}{|l|}{ Serum $A L P, u / l$} \\
\hline Abnormal (High) & $1(33.3)$ & $0.88(0.07-10.00)$ & 0.99 & $3(100)$ & $1.08(0.99-1.18)$ & 0.99 & $0(0)$ & $1.02(0.98-1.08)$ & 0.99 \\
\hline Normal & $12(28.6)$ & & & $39(92.9)$ & & & $1(2.4)$ & & \\
\hline \multicolumn{10}{|l|}{ Serum Albumin, $g / l$} \\
\hline Abnormal (low) & $6(40)$ & $2.17(0.57-8.33)$ & 0.304 & $13(86.7)$ & $0.22(0.02-2.70)$ & 0.254 & $0(0)$ & $0.97(0.89-1.03)$ & 0.99 \\
\hline Normal & $7(23.3)$ & & & $29(96.7)$ & & & $1(3.3)$ & & \\
\hline \multicolumn{10}{|l|}{ Haemoglobin, g/dl } \\
\hline Abnormal (Low) & $8(32)$ & $1.41(0.38-5.26)$ & $0.607^{*}$ & $24(96)$ & $0.38(0.03-4.47)$ & 0.577 & $0(0)$ & $0.95(0.86-1.05)$ & 0.44 \\
\hline Normal & $5(25)$ & & & $18(90)$ & & & $1(5)$ & & \\
\hline \multicolumn{10}{|l|}{ Hbalc, \% } \\
\hline Abnormal (High) & $10(30.3)$ & $1.23(0.29-5.88)$ & 0.99 & $30(90.9)$ & $1.10(0.99-1.22)$ & 0.553 & $1(3)$ & $1.03(0.97-1.10)$ & 0.99 \\
\hline Normal & $3(25)$ & & & $12(100)$ & & & $0(0)$ & & \\
\hline \multicolumn{10}{|l|}{ Serum TC, mmol/l } \\
\hline Abnormal (high) & $1(7.1)$ & $8.19(1.02-71.43)$ & 0.038 & $14(100)$ & $1.11(0.99-1.23)$ & 0.541 & $0(0)$ & $1.03(0.97-1.10)$ & 0.99 \\
\hline Normal & $12(38.7)$ & & & $28(90.3)$ & & & $1(3.2)$ & & \\
\hline
\end{tabular}




\begin{tabular}{|c|c|c|c|c|c|c|c|c|c|}
\hline \multicolumn{10}{|l|}{ Serum TG, mmol/l } \\
\hline Abnormal (high) & $2(9.5)$ & $8.06(1.52-41.67)$ & $0.007^{*}$ & $2(9.5)$ & $0.55(0.05-6.67)$ & 0.99 & $0(0)$ & $1.04(0.96-1.13)$ & 0.99 \\
\hline Normal & $11(45.8)$ & & & $22(91.7)$ & & & $1(4.2)$ & & \\
\hline \multicolumn{10}{|l|}{ Serum HDL, mmol/l } \\
\hline Abnormal (low) & $12(34.3)$ & $0.21(0.02-1.89)$ & 0.238 & $32(91.4)$ & $0.91(0.83-1.01)$ & 0.99 & $1(2.9)$ & $0.97(0.91-1.03)$ & 0.99 \\
\hline Normal & $1(10)$ & & & $10(100)$ & & & $0(0)$ & & \\
\hline \multicolumn{10}{|l|}{ Serum LDL, mmol/l } \\
\hline Abnormal (high) & $6(30)$ & $0.91(0.25-3.33)$ & $0.883 *$ & $19(95)$ & $0.61(0.05-7.14)$ & 0.99 & $0(0)$ & $1.04(0.96-1.12)$ & 0.99 \\
\hline Normal & $7(28)$ & & & $23(92)$ & & & & & \\
\hline
\end{tabular}

$\mathrm{p}<0.05$ = statistically significant; OR, Odds Ratio; CI, Confidence interval; *Chi squared test; PIVKA II, proteins induced by vitamin K absence; PTH, parathyroid hormone; ALP, alkaline phosphatase; TG, triglycerides; TC, total cholesterol; HDL, high

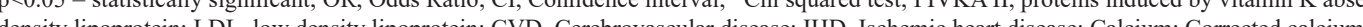


TABLE 4. Independent predictive factor for abnormal vitamin $\mathrm{K}$ level in diabetic patients with chronic kidney disease stage 3-5

\begin{tabular}{lcrc}
\hline Variable & Adjusted odds ratio & 95\% C.I. for & Upper \\
\hline $\begin{array}{l}\text { Phylloquinone }(\mathrm{R} 2=0.38, \mathrm{n}=45) \\
\text { High triglycerides }\end{array}$ & & Lower & 0.66 \\
$\begin{array}{l}\text { PIVKA-II }(\mathrm{R} 2=0.45, \mathrm{n}=45) \\
\text { High PTH }\end{array}$ & 0.112 & 0.02 & 0.017 \\
\hline
\end{tabular}

$\mathrm{p}<0.05$ = statistically significant; PIVKA II, proteins induced by vitamin $\mathrm{K}$ absence; $\mathrm{PTH}$, parathyroid hormone

et al. (2011) reported on recognition of a highly sensitive and reliable HPLC method for the determination of vitamin $\mathrm{K}$ serum concentrations. Immunoassays are characterised by their high specificity and sensitivity, cost effectiveness and capability of detecting the low concentration of residues which makes it useful for routine uses (Zhang et al. 2018). Detection of serum vitamin K1 and PIVKA II using ELISA method has high sensitivity and specificity with no significant cross-reactivity between analogues were observed (Vitamin K1, Amsbio; PIVKA-II, Amsbio). However, there is no study done on development of multianalyte techniques for detection of vitamin $\mathrm{K}$ in a single analytical run. Individuals with DKD have significantly lower phylloquinone concentrations (median 0.53 IQR: 0.72 ) nmol/L (range $0.17-5.12 \mathrm{nmol} / \mathrm{L}$ ) than previously reported individuals with stage 3 to $5 \mathrm{CKD}$ (mean: $2.1 \pm 2.4 \mathrm{nmol} / \mathrm{L}$ range $0-19.3 \mathrm{~mol} / \mathrm{L}$ ) (Holden et al. 2010). DKD patients need more requirement of vitamin $\mathrm{K}$ to form carboxylation of osteocalcin which is necessary and important for glucose metabolism. Previous study by Chen et al. (2013) showed patients with DKD have an imbalance of bone mineral metabolism with reduced level of serum osteocalcin and bone mineral density. We found a strong correlation and association between phylloquinone concentration with total cholesterol and triglycerides. There was a strong independent connection between triglyceride and phylloquinone level. Triacylglycerol-rich lipoproteins transports vitamin $\mathrm{K}$ in the circulation in both postprandial and fasting state (Lamon-Fava et al. 1998). It shows that dietary intake influence circulating phylloquinone which concludes that patients who are clinically well nourished have better vitamin $\mathrm{K}$ status, as reported in previous study (Elliott et al. 2014; Holden et al. 2010). Although it is expected that overall decreased dietary intake and subsequent malnutrition is a possible mechanism for suboptimal vitamin $\mathrm{K}$ status in this population, we could not find any association between serum albumin and phylloquinone at the moment.

Dietary and non-dietary factors affects the phylloquinone concentration. However, in our study, we did not determine non-dietary factors such as apolipoprotein $\mathrm{E}$ (apoE) genotype which is essential for vitamin $\mathrm{K}$ mobilization to peripheral tissues (Newman et al. 2002). High uptake of triglyceride-bound vitamin $\mathrm{K}$ in the liver renders a low vitamin $\mathrm{K}$ delivery to the tissues (Pilkey et al. 2007). The low phylloquinone concentrations observed in our study is postulated as a consequence of low vitamin $\mathrm{K}$ consumption.

PT is a classical method to identify vitamin $\mathrm{K}$ deficiency. PT acts as an indicator of vitamin $\mathrm{K}$ status, however, there must be approximately a $50 \%$ decrease in prothrombin before the prothrombin time becomes abnormal. In our study, only $2.2 \%$ having prolonged PT. Based on this, we can conclude that PT greatly underestimate the actual incidence of vitamin $\mathrm{K}$ deficiency and not a good surrogate marker to identify vitamin K deficiency (Sokoll \& Sadowski 1996). Calcium works together with vitamin $\mathrm{K}$ in clotting cascade. Inadequate calcium level may have impaired haemostasis function. We found similar correlation between PT and corrected calcium, but so far this relationship has not been reported. It has been shown that patients with hypercholesterolemia have non prolonged PT value than patients with lower cholesterol level (Kim et al. 2015). An elevation of procoagulant factors may contribute to short PT value in subjects with raised blood lipids which is implicated in our study.

In this present study, majority of patients $(93.3 \%)$ had elevated PIVKA-II concentrations, confirming that hepatic and peripheral vitamin $\mathrm{K}$ status was not adequate to support the carboxylation of the VKDPs which are osteocalcin and matrix GLA proteins. In our study, higher PIVKA-II concentrations were positively correlated with serum creatinine and PTH levels. It may suggest worsening renal function with low vitamin $\mathrm{K}$ level lead to acceleration of CKD-MBD. In the current study, we observed serum PTH remained significant in multivariable model. The close relationship between high PIVKA II and PTH concentrations may suggest that vitamin $\mathrm{K}$-dependent proteins are important for the regulation of PTH. A study done by Elliott et al. (2014) showed PIVKA II may be a good biomarker of vitamin $\mathrm{K}$ status as its measurement is not influenced by kidney function or dyslipidaemia. Kohlmeier et al. (1997) demonstrated suboptimal vitamin K in haemodialysis patients is associated with increased bone fracture risk and high prevalence of hyperparathyroidism. Strong evidence on the relationship between PIVKA II and PTH in this study showed PIVKA II level has significant effect on CKD-MBD makers. The odds to get abnormal PIVKA II in patients with elevated PTH is 1.99 times more compared to non-elevated level. In addition, our current and previous 
studies have not demonstrated an association between PIVKA II and phylloquinone in patients with CKD stage 3-5 (Elliott et al. 2014).

The relationship between vitamin $\mathrm{K}$ and bone health in CKD non dialysis populations has been studied less intensely, however, a few studies has been published related to vitamin $\mathrm{K}$ and effects on CKD-MBD in end stage renal disease patients. Fusaro et al. (2012) have recently published the results of the vitamin $\mathrm{K}$, vertebral fractures, vascular calcifications and mortality which were an observational study among 387 haemodialysis patients. This study suggests that vitamin $\mathrm{K}$ is important in preserving bone mass and avoiding vascular calcification in haemodialysis patients (Fusaro et al. 2012). Reduced vitamin $\mathrm{K}$ consumption has been associated with low bone mineral density and increased fracture risk in end stage renal disease and treatment with vitamin $\mathrm{K}$ may reduce the risk for hip fractures (Apalset et al. 2011; Evenepoel et al. 2019). Vitamin K supplementation may become an important option in the standard treatment for patients with CKD-MBD complications (Krueger et al. 2009).

\section{CONCLUSION}

In our study we found that vitamin $\mathrm{K}$ deficiency was prevalent in diabetic patients with CKD stage 3-5. Serum triglyceride and serum PTH were independent predictor of Vitamin K deficiency. The main limitation of this study is its cross-sectional nature with small sample size and lack of measurement of other vitamin $\mathrm{K}$ surrogate biomarkers indicating the vitamin $\mathrm{K}$ status, such as dephosphorylated undercarboxylated osteocalcin and matrix GLA protein. The mechanism by which vitamin $\mathrm{K}$ might decrease elevated PTH concentrations and protect against bone fracture in CKD-BMD is still not known. Therefore, future study should be done to study the effect of replacing vitamin $\mathrm{K}$ on CKD-MBD in vitamin $\mathrm{K}$ deficiency CKD patients.

\section{ACKNOWLEDGEMENTS}

This study was funded by grant FF-2018-375 from the Faculty of Medicine, Universiti Kebangsaan Malaysia Medical Centre (UKMMC), Malaysia. We wish to thank staff of the Endocrine laboratories for their technical assistance.

\section{REFERENCES}

Abdulameer, S.A., Sahib, M.N. \& Sulaiman, S.A.S. 2018. The prevalence of osteopenia and osteoporosis among Malaysian Type 2 diabetic patients using quantitative ultrasound densitometer. Open Rheumatology Journal 12: 50-64.

Abougalambou, S.S.I., Abougalambou, A.S. \& Barghash, S.S. 2016. A study evaluating the prevalence of nephropathy among Type 2 diabetes patients attending a teaching hospital in Malaysia. Journal of Clinical Nephrology and Renal Care 2(1): 1-5.
Apalset, E.M., Gjesdal, C.G., Eide, G.E. \& Tell, G.S. 2011. Intake of vitamin $\mathrm{K} 1$ and $\mathrm{K} 2$ and risk of hip fractures: The Hordaland health study. Bone 49(5): 990-995.

Azharuddin, M.K., O'Reilly, D.S., Gray, A. \& Talwar, D. 2007. HPLC method for plasma vitamin K1: Effect of plasma triglyceride and acute-phase response on circulating concentrations. Clinical Chemistry 53(9): 1706-1173.

Belle, M., Brebant, R., Guinet, R. \& Leclercq, M. 1995. Production of a new monoclonal antibody specific to human des-gamma- carboxyprothrombin in the presence of calcium ions. application to the development of a sensitive ELISA-Test. J. Immunoassay. 16(2): 213-229.

Berkner, K.L. \& Runge, K.W. 2004. The physiology of vitamin $\mathrm{K}$ nutriture and vitamin $\mathrm{K}$-dependent protein function in atherosclerosis. Journal of Thrombosis and Haemostasis 2(12): 2118-2132.

Beto, J.A., Fand, R.D.N., Pharmd, W.E.R. \& Bansal, V.K. 2014. Medical nutrition therapy in adults with chronic kidney disease: Integrating evidence and consensus into practice for the generalist registered dietitian nutritionist. Journal of the Academy of Nutrition and Dietetics 114(7): 1077-1087.

Booth, S.L. \& Al Rajabi, A. 2008. Determinants of vitamin K status in humans. Vitamins and Hormones 78: 1-22.

Bujang, M.A., Adnan, T.H., Hashim, N.H., Mohan, K., Kim Liong, A., Ahmad, G., Bak Leong, G., Bavanandan, S. \& Haniff, J. 2017. Forecasting the incidence and prevalence of patients with end-stage renal disease in Malaysia up to the Year 2040. International Journal of Nephrology 2017 : 2735296.

Chen, H., Li, X., Yue, R., Ren, X., Zhang, X. \& Ni, A. 2016. The effects of diabetes mellitus and diabetic nephropathy on bone and mineral metabolism in T2DM patients. Diabetes Research and Clinical Practice 100(2): 272-276.

Cockayne, S., Adamson, J., Lanham-new, S., Shearer, M.J., Gilbody, S. \& Torgerson, D.J. 2006. Vitamin K and the prevention of fractures: Systematic review and metaanalysis of randomized controlled trials. Archives of Internal Medicine 166(12): 1256-1261.

Collins, A., Cashman, K.D. \& Kiely, M. 2006. Phylloquinone (vitamin K1) intakes and serum undercarboxylated osteocalcin levels in Irish postmenopausal women. British Journal of Nutrition 95(5): 982-988.

Cozzolino, M., Ciceri, P., Galassi, A., Mangano, M., Carugo, S., Capelli, I. \& Cianciolo, G. 2019. The key role of phosphate on vascular calcification. Toxins (Basel). 11(4): E213.

Cranenburg, E.C., Schurgers, L.J., Uiterwijk, H.H., Beulens, J.W., Dalmeijer, G.W., Westerhuis, R., Magdeleyns, E.J., Herfs, M., Vermeer, C. \& Laverman, G.D. 2012. Vitamin K intake and status are low in hemodialysis patients. Kidney International 82(5): 605-610.

Elliott, M.J., Booth, S.L., Hopman, W.M. \& Holden, R.M. 2014. Assessment of potential biomarkers of subclinical vitamin $\mathrm{K}$ deficiency in patients with end-stage kidney disease. Canadian Journal of Kidney Health and Disease 1: 13. doi: 10.1186/2054-3581-1-13.

Evenepoel, P., Claes, K., Meijers, B., Laurent, M., Bammens, B., Naesens, M., Sprangers, B., Pottel, H., Cavalier, E. \& Kuypers, D. 2019. Poor vitamin K status is associated with low bone mineral density and increased fracture risk in endstage renal disease. Journal of Bone and Mineral Research 34(2): 262-269.

Forbes, J.M. \& Cooper, M.E. 2013. Mechanisms of diabetic complications. Physiological Reviews 93(1): 137-188. 
Fusaro, M., Noale, M., Viola, V., Galli, F., Tripepi, G., Vajente, N., Plebani, M., Zaninotto, M., Guglielmi, G., Miotto, D., Dalle Carbonare, L., D’Angelo, A., Naso, A., Grimaldi, C., Miozzo, D., Giannini, S. \& Gallieni, M. 2012. Vitamin K, vertebral fractures, vascular calcifications, and mortality: Vitamin K Italian (VIKI) dialysis study. Journal of Bone and Mineral Research 27(11): 2271-2278.

Gallieni, M. \& Fusaro, M. 2014. Vitamin K and cardiovascular calcification in CKD: Is patient supplementation on the horizon? Kidney International 86(2): 232-234.

Ghaderian, S.B., Hayati, F., Shayanpour, S. \& Beladi Mousavi, S.S. 2015. Diabetes and end-stage renal disease: A review article on new concepts. J. Renal. Inj. Prev. 4(2): 28-33.

Goh, B.L., Ong, L.M. \& Lim, Y.N. 2014. 21st Report of the Malaysian Dialysis \& Transplant Registry 2013. Kuala Lumpur: The National Renal Registry Malaysia: https:// doi.org/10.1143/JJAP.35.L657.

Holden, R.M., Morton, A.R., Garland, J.S., Pavlov, A., Day, A.G. \& Booth, S.L. 2010. Vitamins K and D status in stages 3-5 chronic kidney disease. Clinical Journal of the American Society of Nephrology 5(4): 590-597.

Ikeda, Y., Iki, M., Morita, A., Kajita, E., Kagamimori, S., Kagawa, Y. \& Yoneshima, H. 2006. Nutritional epidemiology intake of fermented soybeans, natto, is associated with reduced bone loss in postmenopausal women: Japanese populationbased osteoporosis (JPOS) study. Journal of Nutrition 136(5): 1323-1328.

Kaesler, N., Magdeleyns, E., Herfs, M., Schettgen, T., Brandenburg, V., Fliser, D., Vermeer, C., Floege, J., Schlieper, G. \& Krüger, T. 2014. Impaired Vitamin K recycling in uremia is rescued by Vitamin K supplementation. Kidney Int. 86(2): 286-293.

Khir, A.S., Pheng, A.C.S., Endocrinologist, C., Panel, E., Abdullah, A.B.J., Radiologist, C. \& Surgeon, C.O. 2012. Clinical practice guidelines on management of osteoporosis. Osteoporosis 2012: 1-53.

Kidney Disease: Improving Global Outcomes (KDIGO) CKD-MBD Work Group. 2009. KDIGO clinical practice guideline for the diagnosis, evaluation, prevention, and treatment of Chronic Kidney Disease-Mineral and Bone Disorder (CKD-MBD). Kidney International Suppl. 113: S1-130.

Kim, J.A., Kim, J.E., Song, S.H. \& Kim, H.K. 2015. Influence of blood lipids on global coagulation test results. Ann. Lab. Med. 35(1): 15-21.

Klawansky, S., Komaroff, E., Cavanaugh Jr., P.F., Mitchell, D.Y., Gordon, M.J., Connelly, J.E. \& Ross, S.D. 2003. Relationship between age, renal function and bone mineral density in the US population. Osteoporosis International 14(7): 570-576.

Kohlmeier, M., Saupe, J., Shearer, M.J., Schaefer, K. \& Asmus, G. 1997. Bone health of adult hemodialysis patients is related to vitamin $\mathrm{K}$ status. Kidney International 51(4): 1218-1221.

Krueger, T., Westenfeld, R., Ketteler, M., Schurgers, L.J. \& Floege, J. 2009. Vitamin K deficiency in CKD patients: A modifiable risk factor for vascular calcification? Kidney International 76(1): 18-22.

Lamon-Fava, S., Sadowski, J.A., Davidson, K.W., O’Brien, M.E., McNamara, J.R. \& Schaefer, E.J. 1998. Plasma lipoproteins as carriers of phylloquinone (Vitamin K1) in humans. The American Journal of Clinical Nutrition 67(6): 1226-1231.
Larsson, S.C., Traylor, M. \& Markus, H.S. 2018. Circulating vitamin $\mathrm{K} 1$ levels in relation to ischemic stroke and its subtypes: A mendelian randomization study. Nutrients 10(11): E1575.

Levey, A.S., Stevens, L.A., Schmid, C.H., Zhang, Y.L., Castro, A.F., Feldman, H.I., Kusek, J.W., Eggers, P., Van Lente, F., Greene, T. \& Coresh, J. 2009. A new equation to estimate glomerular filtration rate. Annals. of Internal Medicine 150(9): 604-612.

Marinova, M., Lütjohann, D., Westhofen, P., Watzka, M., Breuer, O. \& Oldenburg, J.A. 2011. Validated HPLC method for the determination of vitamin $\mathrm{K}$ in human serum - First application in a pharmacological study. The Open Clinical Chemistry Journal 4: 17-27.

McCabe, K.M., Adams, M.A. \& Holden, R.M. 2013. Vitamin $\mathrm{K}$ status in chronic kidney disease. Nutrients 5(11): 43904398.

Newman, P., Bonello, F., Wierzbicki, A.S., Lumb, P., Savidge, G.F. \& Shearer, M.J. 2002. The uptake of lipoprotein-borne phylloquinone (Vitamin K1) by osteoblasts and osteoblastlike cells: Role of heparan sulfate proteoglycans and apolipoprotein E. Journal of Bone and Mineral Research 17(3): 426-433.

Pilkey, R.M., Morton, A.R., Boffa, M.B., Noordhof, C., Day, A.G., Su, Y., Miller, L.M., Koschinsky, M.L. \& Booth, S.L. 2007. Subclinical Vitamin K deficiency in hemodialysis patients. American Journal of Kidney Diseases 49(3): 432439.

Pillai, A., Eranki, V., Shenoy, R. \& Hadidi, M. 2011. Age related incidence and early outcomes of hip fractures: A prospective cohort study of 1177 patients. Journal of Orthopaedic Surgery and Research 6: 5. https://doi.org/10.1186/1749799X-6-5.

Quick, A.J. 1975. The role of vitamins in hemostasis. Thromb. Diath. Haemorrh. 33(2): 191-198.

Sokoll, L.J. \& Sadowski, J.A. 1996. Comparison of biochemical indexes for assessing status in a healthy adult population. American Journal of Clinical Nutrition 63(4): 566-573.

Tsugawa, N., Shiraki, M., Suhara, Y., Kamao, M., Tanaka, K. \& Okano, T. 2006. Vitamin K status of healthy Japanese women: Age-related vitamin K requirement for gammacarboxylation of osteocalcin. American Journal of Clinical Nutrition 83(2): 380-386.

Vissers, L.E., Dalmeijer, G.W., Boer, J.M., Monique Verschuren, W.M., van der Schouw, Y.T. \& Beulens, J.W. 2013. Intake of dietary phylloquinone and menaquinones and risk of stroke. Journal of the American Heart Association 2(6): e000455.

van Ballegooijen, A.J., Cepelis, A., Visser, M., Brouwer, I.A., van Schoor, N.M. \& Beulens, J.W. 2017. Joint association of low Vitamin D and Vitamin K status with blood pressure and hypertension. Hypertension 69(6): 1165-1172.

Yan, L., Zhou, B., Greenberg, D., Wang, L., Nigdikar, S., Prynne, C. \& Prentice, A. 2004. Vitamin K status of older individuals in Northern China is superior to that of older individuals in the UK. British Journal of Nutrition 92(6): 939-945.

Zhang, Y., Zhou, W.E., Yan, J.Q., Liu, M., Zhou, Y., Shen, X., Ma, Y.L., Feng, X.S., Yang, J. \& Li, G.H. 2018. A review of the extraction and determination methods of thirteen essential vitamins to the human body: An update from 2010 Molecules 23(6): E1484. 
S. Jayakumar, Arbaiyah Bain \& Abdul Halim Abdul Gafor* Nephrology Unit, Department of Medicine

Universiti Kebangsaan Malaysia Medical Centre

Jalan Yaacob Latif, Bandar Tun Razak

Cheras, 56000 Kuala Lumpur, Federal Territory

Malaysia

Elena Azizan

Department of Medicine

Universiti Kebangsaan Malaysia Medical Centre

Jalan Yaacob Latif, Bandar Tun Razak

Cheras, 56000 Kuala Lumpur, Federal Territory

Malaysia
Shamsul Azhar Shah

Department of Community Health

UKM Medical Molecular Biology Institute

Jalan Yaacob Latif, Bandar Tun Razak

Cheras, 56000 Kuala Lumpur, Federal Territory

Malaysia

*Corresponding author; email: halimgafor@gmail.com

Received: 9 October 2019

Accepted: 13 November 2019 